Research Paper

\title{
Granzyme family acts as a predict biomarker in cutaneous melanoma and indicates more benefit from anti-PD-1 immunotherapy
}

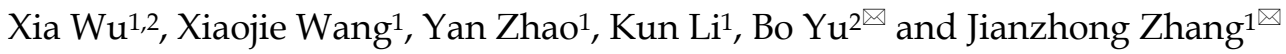 \\ 1. Department of Dermatology, Peking University People's Hospital, Beijing 100044, China. \\ 2. Department of Dermatology, Skin Research Institute of Peking University Shenzhen Hospital, Peking University Shenzhen Hospital, Shenzhen, 518036, \\ China. \\ $\bowtie$ Corresponding authors: E-mail: yubomd@163.com (Bo Yu); rmzjz@126.com (Jianzhong Zhang).
}

(c) The author(s). This is an open access article distributed under the terms of the Creative Commons Attribution License (https://creativecommons.org/licenses/by/4.0/). See http:/ /ivyspring.com/terms for full terms and conditions.

Received: 2020.10.20; Accepted: 2021.01.06; Published: 2021.02.06

\begin{abstract}
The incidence of cutaneous melanoma (CM) increased since the 1970s, and also along with an unfavorable prognosis. CM patients have been verified benefits from immunotherapy, and granzymes (GZMs) comprise more than $90 \%$ of the cytolytic granules secreted by cytotoxic T lymphocytes and nature killer cell. Therefore, it is essential to evaluate the prognostic value of GZMs in CM. A total of 633 CM patients was enrolled to access the prognostic value of GZMs. The integrated prognostic value of five GZMs was validated in TCGA-SKCM, GSE65904, GSE53118, GSE19234 and GSE22153 cohorts. GZMscore, age, Breslow's depth and tumor stage are the independent risk factors for CM patients, risk score based on these factors was calculated in TCGA-SKCM and GSE65906 cohorts, which could polarize the CM patients to high- and low-risk groups with diverse prognosis. Patients in low-risk group obtained the activated immune signaling pathways and response, especially for the activated CD8+ T cells, and could benefit more from anti-PD-1 therapy. A higher tumor mutation burden was observed in low-risk group, especially for the mutation of BRAF. The protect function of GZMK was confirmed by CM cell lines, overexpression of GZMK in A375 and G361 cells suppresses cell proliferation, migration, but not cell apoptosis. All in all, we revealed the prognostic value of GZMs in CM patients, which could also act as a predicted value for the selection of responders of anti-PD-1 immunotherapy.
\end{abstract}

Key words: granzymes; cutaneous melanoma; overall survival; risk score

\section{Introduction}

Melanoma is a kind of malignant skin tumor consists of the pigment-producing cells, which could format on other organs, including eyes, ears, gastrointestinal tract, oral and genital mucous membranes [1]. Cutaneous melanoma (CM) only accounts for less than $5 \%$ of all skin cancers, but caused a greater number of skin cancer-specific deaths [2]. There are about 287,723 new cases and 60,712 specific death of $\mathrm{CM}$ around the world [3]. The non-melanoma cancer of skin accounts for $5.8 \%$ of all new tumors, and leads to only $0.7 \%$ deaths, while the $\mathrm{CM}$ only accounts for $1.6 \%$ of all tumor cases, but accounts for about $0.6 \%$ deaths [3]. In the past decades, the mortality of $\mathrm{CM}$ increased sharply, the median mortality of per ten thousand men increased from 1.55 to 2.57 , while the mortality of per ten thousand women increased from 1.39 to 1.55 [4]. The tumorigenesis of $\mathrm{CM}$ is affected by both genetic background, including family history and DNA mutations, and environmental factors, including fair-skinned and light-haired persons with high sunburn susceptibility, increased exposure to ultraviolet radiation (UV-A and UV-B rays), and arsenic [5-7]. The incidence of CM has increased since the early 1970s in predominantly fair-skinned populations [1]. The overall survival (OS) is less to 6 month for the advanced stage of CM patients, and improved since 2011, with the successful treatment of CM with BRAF inhibitor, anti-CTLA-4 therapy, and anti-PD-1 therapy, with a favorable OS result of about 
$40 \%$ 5-year survival rate [8-11]. However, only part of the patients could benefit from the above-mentioned therapy, it is necessary to reveal the new biomarkers for the prediction of prognosis and treatment.

Granzymes are a family of homologous serine proteases, which could induce the apoptosis of virus-infected cells or tumor cells accompanied by the perforin [12]. Granzymes comprise more than $90 \%$ of the cytolytic granules secreted by cytotoxic $\mathrm{T}$ lymphocytes and nature killer (NK) cells [13, 14]. Immunocytes are the pivotal components for the tumor immune microenvironment, which was also reported as the prognostic marker for cancer [15-17]. There are five granzymes $(\mathrm{A}, \mathrm{B}, \mathrm{H}, \mathrm{K}, \mathrm{M})$ in humans, each of them contains the specific substrate and executes the different pathways to the function of promoting cell death $[18,19]$. However, the total value of granzymes family (GZMs) in CM is not clear till to now. In the current study, we evaluated the prognostic value of GZMs in TCGA-SKCM cohort; the prognostic signature was also established and validated in GSE65904 cohort. The potential GZMs impacted signaling pathways, gene mutation, and response to immunotherapy was also extracted.

\section{Materials and methods}

\section{Patients and datasets}

A total of 507 patients diagnosed with $\mathrm{CM}$ was enrolled for the current study, 471 of them from The Cancer Genome Atlas (TCGA)-CM cohort, while the other 216 extracted from GSE65904, GSE53118, GSE19234 and GSE22153 cohorts. The mRNA expression profile, gene somatic mutation data, as well as the matched clinical information of TCGA-CM cohort was downloaded from UCSC Xena (https:/ / tcga.xenahubs.net). The clinical information and expression data of GSE65904, GSE53118, GSE19234 and GSE22153 cohorts were obtained from the Gene Expression Omnibus database (https://www.ncbi. nlm.nih.gov/geo/).

\section{Construction and confirm of the GZMs predicting signature}

To combine the effectiveness of five GZMs to $\mathrm{CM}$, we employed the single-sample Gene Set Enrichment Analysis (ssGSEA) analysis, which conducted by the R Bioconductor package Gene Set Variation Analysis (GSVA, v.3.5), to define the ssGSEA GZMs socre (GZMscore) representing the degree of absolute enrichment of the five GZMs in each sample. The GZMscore and other major clinical parameters of $\mathrm{CM}$ patients were all enrolled to construct the overall survival (OS) predicting model by multivariate Cox regression analysis. The coefficient of each parameter generated by the multivariate Cox was used to calculate the risk score,

$$
\text { Riskscore }=\sum_{i=1}^{n} \text { Parameter }_{i}
$$

Parameter $_{i}$ was the value of $i^{\text {th }}$ parameter. Heatmap was used to show the distribution of each parameter of each patient in the overall cohort. K-M survival analysis was used to indicate the different outcomes of risk groups with the 'survminer' package. The predicting value of the risk score to $\mathrm{CM}$ was also confirmed in the GSE65904 cohort.

\section{Different expression genes (DEGs), gene enrichment annotation and Gene Set Enrichment Analysis (GSEA) analysis}

The DEGs between GZMs determined high- and low-risk groups were captured by the "limma" package", the cut-off value was applied with the gene expression fold-change $>1$ and $P$ value $<0.05$. And then, we annotated the DEGs to reveal the activated key signaling pathways Metascape (http:// metascape.org) [20]. We also conducted GSEA analysis based on the gene expression microarray data, which could detect the significant biological differences between different groups [21]. The pathway analyses of training and validation cohorts were performed separately. The enrichment score (ES) is the maximum deviation from zero encountered during that walk, which reflects the degree to which the genes in a gene set are overrepresented at the top or bottom of the entire ranked list of genes. The resulting normalized enrichment scores (NES) is used to adjust the analysis results across different gene sets. The false discovery rate (FDR) q-values were used to adjust for multiple hypothesis testing.

\section{Tumor immune microenvironment, tumor mutation burden (TMB) and response to immunotherapy in $C M$ patients}

The infiltration of 28 immunocytes was assessed by the ssGSEA with the 28 gene sets of immune cell markers, which were previously reported by Charoentong et al. [22]. The mutation landscape Oncoprint was drawn by R package "maftools" [23]. The TMB was also calculated by the "maftools". To evaluate the individual likelihood of responding to immunotherapy, we employed the Tumor Immune Dysfunction and Exclusion (TIDE) algorithm [24-26]. Further, based on a CM cohort that receive anti-CTLA-4 or anti-PD-1 checkpoint inhibition therapy, the specific gene sets were obtained, with 795 genes [27]. Subclass mapping analysis was conducted to compare the similarity of the risk groups with the immunotherapy subgroups, aim to point out the 
responders of anti-CTLA-4 or anti-PD-1 immunotherapy [24].

\section{Cell lines and cell culture}

The melanoma cell lines (A375 and G361) were purchased from the Institute of Cell Research, Chinese Academy of Sciences, Shanghai, China. The A375 and G361 cells were maintained in 1640 medium (Invitrogen, Carlsbad, CA, USA) with 10\% fetal bovine serum (FBS) and 1\% antibiotics $(100 \mathrm{ug} / \mathrm{ml}$ streptomycin and $100 \mathrm{U} / \mathrm{ml}$ penicillin) at $37^{\circ} \mathrm{C}$ in the atmosphere of $5 \% \mathrm{CO}_{2}$.

\section{Construction of GZMK overexpression vector and Cell transfection}

The GZMK overexpression vector was constructed by Syngentech Co., Ltd. (Beijing, China). Cells were transfected with vectors using Lipofectamine 3000 Transfection Reagent (Invitrogen, Carlsbad, CA, USA) according to the manufacturer.

\section{RNA extraction and qRT-PCR}

TRIZOL (Invitrogen, Grand Island, NY, USA) was used to extract the total RNA from cells, PrimeScript RT Kit with a gDNA Eraser (Takara, Dalian, China) was employed to synthesize the cDNA. Roche light cycler 480 Real-Time PCR machine with the SYBRs Premix Ex Taq (Takara, Dalian, China) was used to perform the Quantitative real-time PCR (qRT-PCR) analysis. The primers were synthesized by Sangon Biotech (Shanghai, China), with the sequence of: GZMK forward: ATGCTGGTTA AGCTTCAAACAG, GZMK-reverse: GCATTTGGTTC CAGATCTAAGAG; GAPDH-forward: CGCTCTCTG CTCCTCCTGTTC, GAPDH-reverse: ATCCGTTGACT CCGACCTTCAC. The expression of GZMK in different groups was evaluated by the $\Delta \mathrm{Ct}$ value.

\section{Wound healing assay}

The migration ability of CM cell lines, A375 and G361 was evaluated by the wound healing assay. Cells were pre-seeded in the 6-well plate to get more than $90 \%$ confluence and serum-starved for 24 hours. We scratched the wounds by the sterile $200 \mu$ pipette tips. The wounds were recorded after the infliction of 0 hours, and 24 hours. The quantified results of the wound healing assay were calculated by the covered area to compare to the all wound area.

\section{Cell proliferation assay}

We compared the cell proliferation between GZMK overexpression infected group and negative control vector group by cell counting kit (CCK-8) assay (Transgen, Beijing, China). $3 \times 10^{3}$ cells were seeded in per well in a 96-well plate, at the end time of $0 \mathrm{~h}, 24 \mathrm{~h}, 48 \mathrm{~h}$, and $72 \mathrm{~h}, 10 \mu \mathrm{l}$ of CCK- 8 solution was added to each well and incubated with 2 hours. The reader machine (Bio-Rad, Hercules, CA, USA) was then used to assess the absorbance at $450 \mathrm{~nm}$. Colony formation assay was also employed to evaluate cell proliferation affected by GZMK overexpression. 2000 cells/well infected by GZMK vector or NC vector were pre-seeded in $6 \mathrm{~cm}$ dishes and incubated for ten days. At the end of the assay, $0.1 \%$ crystal violet was added to stain the cells, and then measured the absorbance of $550 \mathrm{~nm}$ using a microplate reader.

\section{Flow cytometry assay}

G361 and A375 cells were seeded in 6-well plates. $24 \mathrm{H}$ after transfection, the transfected cells were harvested using trypsin without EDTA. According to the manufacturer's protocols, the FITC Annexin V Apoptosis Detection Kit (TransGen, Beijing, China) could double stain cells with FITC-Annexin and PI. The ratio of early apoptotic cells in melanoma cells was determined by the flow cytometry (EPICS, XL-4, Beckman, CA, USA). The experiments were done at least three times.

\section{Statistical methods and online tools}

K-M survival analysis was used to indicate the different clinical outcomes of subgroups with the 'survminer' package, univariate Cox regression analysis was employed to calculate the hazard ration (HR) and 95\% confidence interval (95\% CI). Comparisons of continuous data between two groups were performed by the Student's T-test or Wilcoxon test. Pearson correlation coefficient test was employed to assess the relationship between two factors. The distributions of categorical variable between highand low-risk groups were compared by the Chi-square test. For in vitro experiments, all data from at least three independent experiments were presented as mean \pm standard deviation (SD). All statistical data were analyzed by SPSS 20.0 software (SPSS Inc. Chicago, IL, USA). A two-sided P value < 0.05 was considered statistically significant. All analyses were performed by $\mathrm{R}$ version 3.6.5 (http://www.r-project.org). The prognostic value of GZMs in CM patients was also evaluated by GEPIA (http://gepia.cancer-pku.cn/index.html),

PROGgeneV2 (http://genomics.jefferson.edu/ proggene/index.php) [28] and SurvExpress (http:/ / bioinformatica.mty.itesm.mx:8080/Biomatec /SurvivaX.jsp) [29]. The infiltration of immunocytes in wild type or mutated gene subgroup was obtained from the TIMER 2.0 (http:/ / timer.cistrome.org/) [30]. 


\section{Results}

\section{GZMs act as the protectors in CM progression}

We first evaluated the prognostic value of GZMs in TCGA-SKCM cohort. The median expression value of each gene used as the cut-off value to divide patient to high expression and low expression groups. We generated the results that low expression of GZMA $(\mathrm{HR}=0.54,95 \% \mathrm{CI}=0.417-0.712, \mathrm{P}<0.001), \mathrm{GZMB}$ $(\mathrm{HR}=0.53,95 \% \mathrm{CI}=0.404-0.694, \mathrm{P}<0.001), \mathrm{GZMH}$ $(\mathrm{HR}=0.55,95 \% \mathrm{CI}=0.420-0.719, \mathrm{P}<0.001), \mathrm{GZMK}$ $(\mathrm{HR}=0.57,95 \% \mathrm{CI}=0.436-0.745, \mathrm{P}<0.001)$ and GZMM $(\mathrm{HR}=0.76,95 \% \mathrm{CI}=0.580-0.987, \mathrm{P}<0.001)$ indicate unfavorable prognosis (Figure 1A-E). We also observed the decreased expression of GZMs in
$\mathrm{CM}$ with the Brelow's depth high than $3 \mathrm{~cm}$, which also link the decreased expression of GZMs with advanced CM (all, $\mathrm{P}<0.05$, Figure 1F). The combined prognostic value of GZMs was evaluated by online tools, GEPIA ( $\mathrm{HR}=0.57, \mathrm{P}<0.001$, Figure 1G), and SurvExpress $(\mathrm{HR}=1.87,95 \% \mathrm{CI}=1.38-2.53, \mathrm{P}<0.001$, Figure 1H). We also used the PROGgeneV2 to predict the combined prognostic value of GZMs on TCGA-SKCM cohort $(\mathrm{HR}=0.88,95 \% \mathrm{CI}=0.81-0.97, \mathrm{P}$ $=0.008$, Figure 1I), GSE53118 cohort $(\mathrm{HR}=0.61,95 \%$ $\mathrm{CI}=0.43-0.89, \mathrm{P}=0.010$, Figure S2), GSE19234 cohort $(\mathrm{HR}=0.59,95 \% \mathrm{CI}=0.36-0.97, \mathrm{P}=0.036$, Figure S3 $)$, GSE22153 cohort $(\mathrm{HR}=0.66,95 \% \mathrm{CI}=0.49-0.90, \mathrm{P}=$ 0.008 , Figure S4). These results confirmed the new findings about GZMs to CM prognosis.
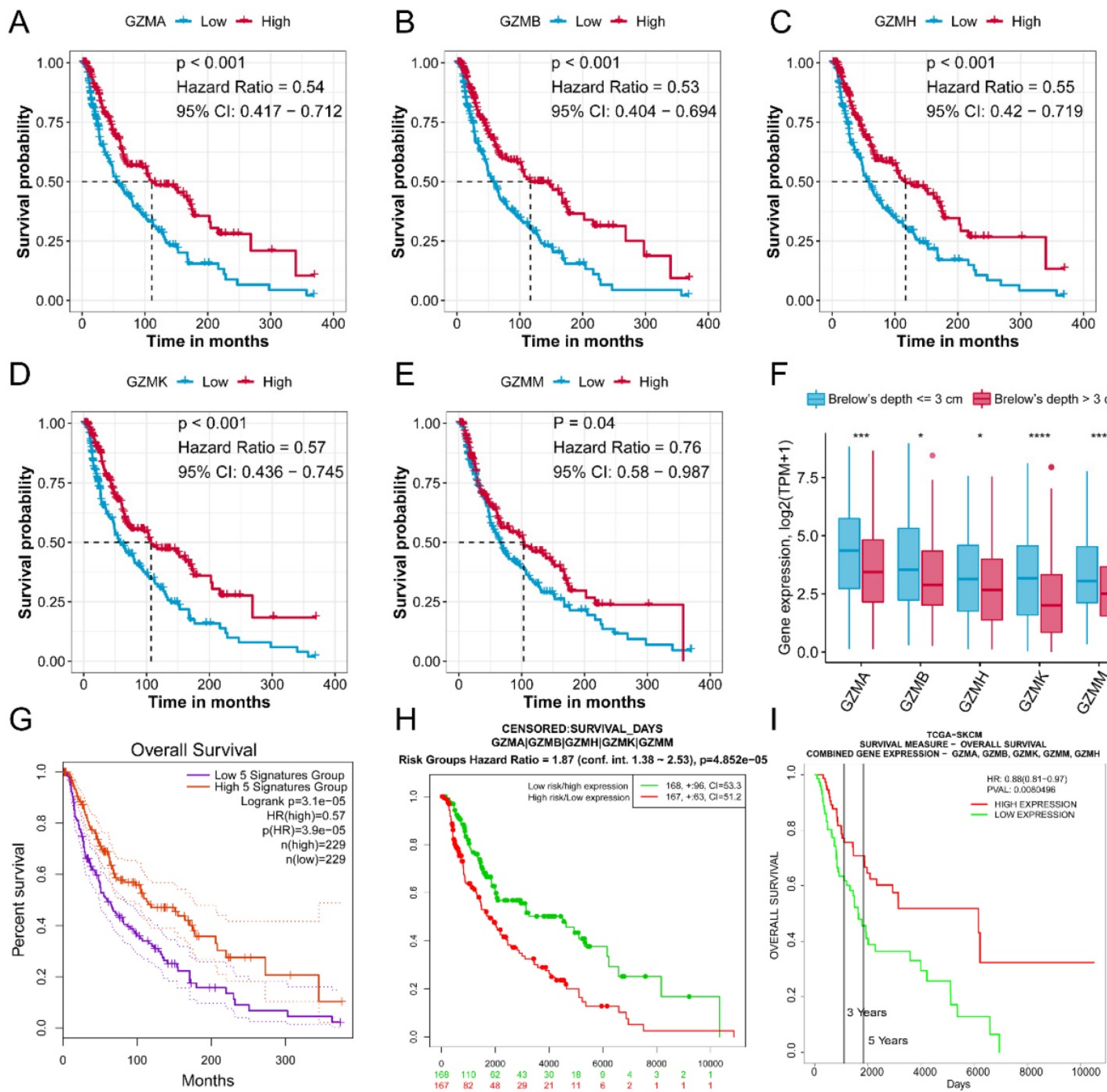

$\mathrm{F}$
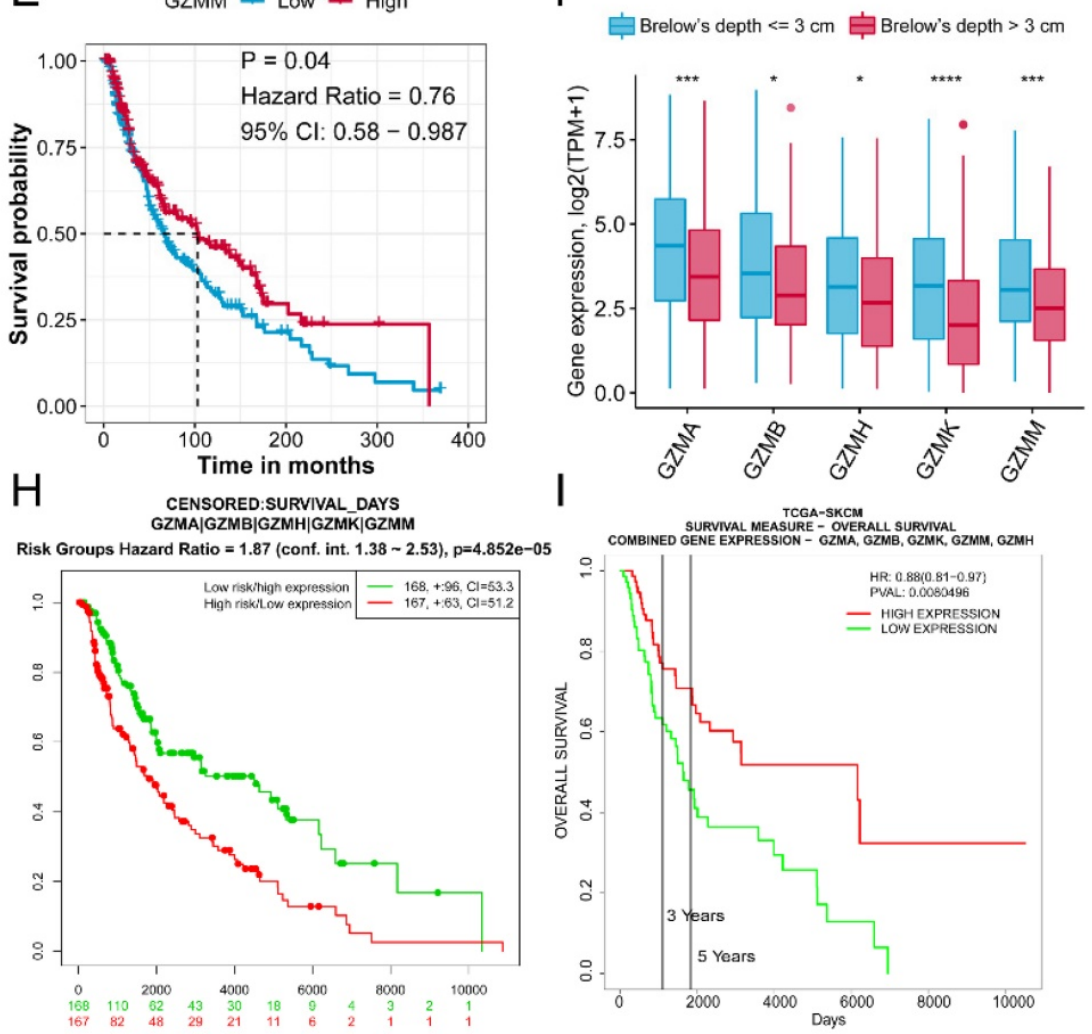

Figure 1. GZMs are prognostic factors for CM patients. Low expression of GZMA (A), GZMB (B), GZMH (C), GZMK (D), GZMM (E) indicate poor prognosis for CM patients; (F) Lower expression of GZMs was observed in patients with CM Brelow's depth higher than 3 cm; The combined prognostic value of GZMs validated with GEPIA (G), PROGgeneV2 (H), and SurvExpress (l). 

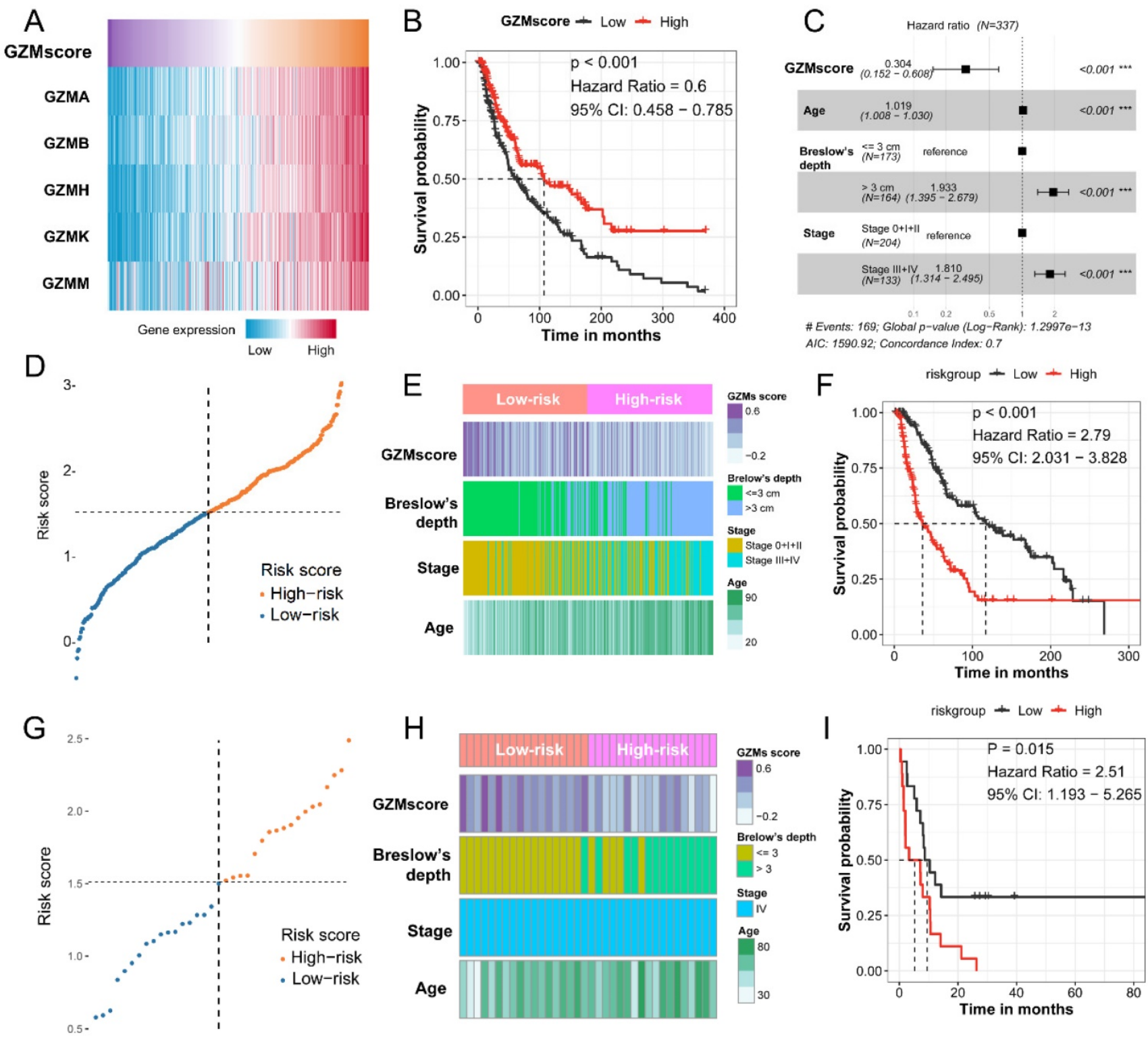

Figure 2. GZMs and clinical feature determined risk score could predict the prognosis of CM patients. (A) GZMscore calculated by the expression of five GZMs with ssGSEA; (B) GZMscore could separate the CM patients to favorable and poor prognosis; (C) Multivariate Cox analysis showing the prognostic value of GZMscore, age, Breslow's depth and stage; (D) Risk score calculated by the formula in TCGA-SKCM cohort; (E) Distribution of GZMscore, age, Breslow's depth and stage in high- and low-risk groups of TCGA-SKCM cohort; (F) K-M plot showing the different prognosis in high- and low-risk groups of TCGA-SKCM cohort; (G) Risk score calculated by the formula in GSE65904 cohort; (H) Distribution of GZMscore, age, Breslow's depth and stage in high- and low-risk groups of GSE65904 cohort; (I) K-M plot showing the different prognosis in high- and low-risk groups of GSE65904 cohort.

Table 1. The predicted value of GZMscore and clinical features to CM patients

\begin{tabular}{|c|c|c|c|c|c|c|}
\hline \multirow[t]{2}{*}{ Parameters } & \multicolumn{3}{|c|}{ Univariate Cox Analysis } & \multicolumn{3}{|c|}{$\begin{array}{l}\text { Multivariate Cox } \\
\text { Analysis }\end{array}$} \\
\hline & $\overline{\mathrm{HR}}$ & $95 \% \mathrm{CI}$ & $P$ value & HR & $95 \% \mathrm{CI}$ & $P$ value \\
\hline GZMscore & 0.6 & $0.46-0.79$ & $2.00 \times 10^{-4^{*}}$ & 0.3 & $0.15-0.61$ & $7.62 \times 10^{-4}$ \\
\hline Age & 1.02 & $1.01-1.03$ & $2.28 \times 10^{-5^{*}}$ & 1.02 & $1.01-1.03$ & $9.11 \times 10^{-4}$ \\
\hline $\begin{array}{l}\text { Gender } \\
\text { (Male vs. Female) }\end{array}$ & 0.944 & $0.69-1.30$ & 0.721 & & & \\
\hline $\begin{array}{l}\text { Brelow's depth } \\
(>3 \mathrm{~cm} \text { vs. } \leq 3 \mathrm{~cm})\end{array}$ & 2.49 & $1.82-3.42$ & $1.53 \times 10^{-8^{*}}$ & 1.95 & $1.40-2.71$ & $7.42 \times 10^{-5}$ \\
\hline $\begin{array}{l}\text { Stage } \\
(\mathrm{III}+\mathrm{IV} \text { vs. } 0+\mathrm{I}+\mathrm{II})\end{array}$ & 2.01 & $1.47-2.76$ & $1.39 \times 10^{-5^{*}}$ & 1.81 & $1.31-2.49$ & $2.86 \times 10^{-4}$ \\
\hline
\end{tabular}

\section{GZMs determined signature acts well to predict the prognosis of $C M$ patients}

To combined the value of five GZMs expression, we used ssGSEA to generate the GZMscore (Figure 2A), lower GZMscore of GZMs predict the poor prognosis of $\mathrm{CM}$ patients $(\mathrm{HR}=0.6,95 \% \mathrm{CI}=$ 0.458-0.785, $\mathrm{P}<0.001$, Figure 2B), which consist with the results in Figure 1. With the help of univariate and multivariate Cox regression analysis, we revealed that the GZMscore, Age, Breslow's depth, and tumor stage are the independent risk factors for the prognosis of $\mathrm{CM}$ patients (Table 1, Figure 2C), the coefficient of each factor was also obtained (Table 2). Based on the 
formula mentioned in the method, we calculated the risk score of each patient in TCGA-SKCM cohort, and separated into high- and low-risk groups (Table 3, Figure 2D). We observed the high-risk patients contained a lower GZMsocre, more tumors with Breslow's depth, advanced stage (stage III + IV) and higher age (Figure 2E), as well as the unfavorable overall survival $(\mathrm{HR}=2.79,95 \% \mathrm{CI}=2.031-3.828, \mathrm{P}<$ 0.001 , Figure 2F). To validate the accuracy and stability of the signature, we calculated the risk score of the 36 patients from GSE65904 (Figure 2G). The similar tendency reappeared in GSE65904 cohort. Patients in high-risk group shown a lower GZMscore, more with Brelow's depth, and older age (Figure 2H). Also, the high-risk group patients linked with poor prognosis of $\mathrm{CM}$ patients $(\mathrm{HR}=2.51,95 \% \mathrm{CI}=$ 1.193-5.265, $\mathrm{P}=0.015$, Figure $2 \mathrm{I})$.

\section{Overexpressed of GZMK could inhibit cell proliferation, migration, but not apoptosis}

Based on the bioinformatics analysis, we revealed that the GZMK acts as a gene suppressor in $\mathrm{CM}$. To validate the function of GZMK in cell lines, we first constructed the GZMK overexpressed CM cell lines, the overexpressed GZMK was detected by PCR and qRT-PCR in A375 and G361 CM cell lines (Figure S1A-B). As compared with the negative control (NC) group, the fold proliferation rate of decreased in GZMK overexpression group in A375 and G361 cell lines $(\mathrm{P}<0.05$, Figure 3A-B). Colony formation assay
A

A375

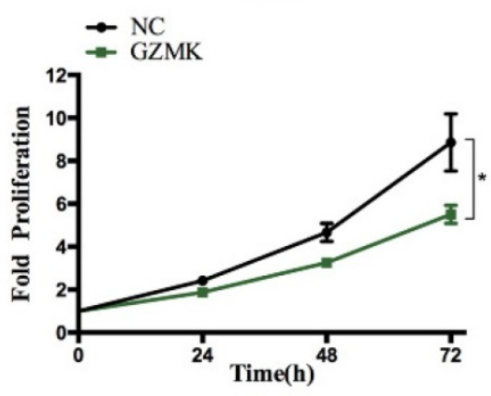

C
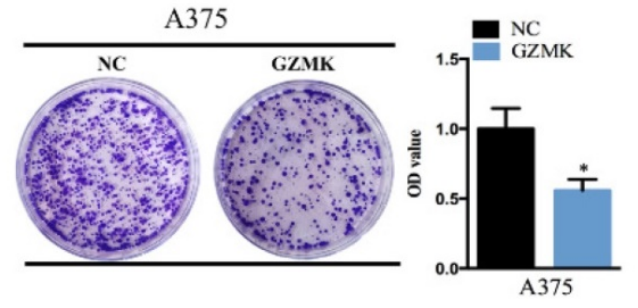

E
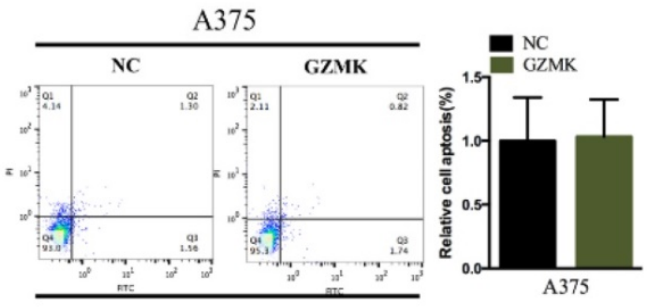

G
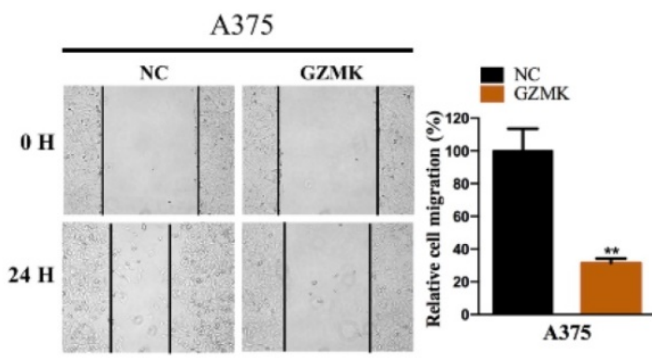

B

G361

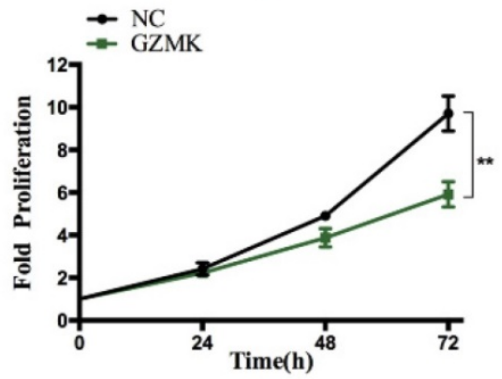

$\mathrm{D}$
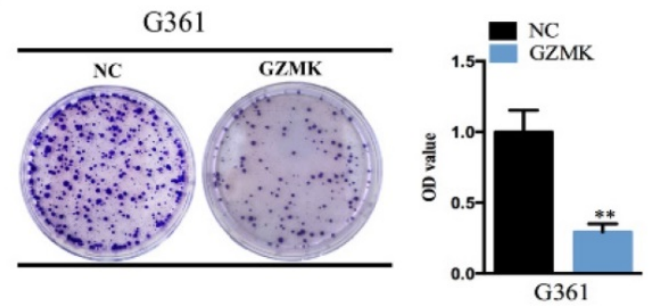

$\mathrm{F}$
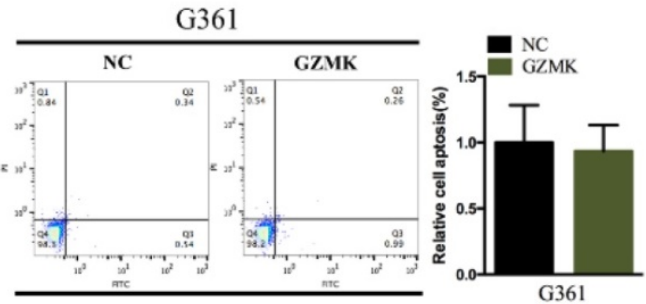

$\mathrm{H}$

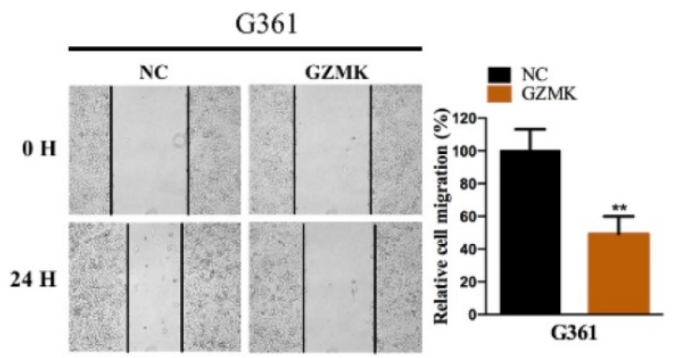

Figure 3. Overexpression of GZMK suppressed the cell proliferation and migration of CM cells. Overexpression of GZMK suppressed cell proliferation detected by CCK-8 assay and colony-formation assay in A375 (A, C) and G361 (B, D) cell line; Overexpression of GZMK didn't impact cell apoptosis (E-F); Overexpression of GZMK suppressed cell migration detected by wound healing assay $(G-H)$. 
is another way to evaluate the impact of GZMK on cell proliferation, the OD value of GZMK overexpression group significantly lower than that in NC group $(\mathrm{P}<$ 0.05, Figure 3C-D). As to the effect on cell apoptosis, we didn't revealed the association between GZMK and cell apoptosis, the ratio of early apoptotic cells in NC group and GZMK group shown a similar distribution in both A375 and G361 cell lines (Figure 3E-F). We evaluated the alteration of cell migration after overexpression of GZMK, as compared with the 0-hour control, cell migration less in GZMK group than NC group $(\mathrm{P}<0.05$, Figure $3 \mathrm{G}-\mathrm{H})$. Based on the results generated from in vitro cell experiment, we could obtain the conclusion that the GZMK could inhibit $\mathrm{CM}$ cell proliferation, migration, but not cell apoptosis, to inhibit the tumorigenesis of $\mathrm{CM}$.

\section{Different activated signaling pathways among GZMs determined high- and low- risk groups}

To further understand the mechanism of how GZMs impact the tumorigenesis of CM, we extracted the DEGs between high- and low-risk groups of CM patients in TCGA-SKCM cohort. With the cut-off value of $P$ value less than 0.05 , and $\log 2$ (Fold Change) less than -1 or high than 1 , a total of 611 decreased
DEGs was revealed among the risk groups, as well as another 170 DEGs was observed increasing in high-risk group (Figure 4A). The annotation results uncovered that the 611 DEGs enriched in several immune associated signaling pathways, including the biological process of leukocyte migration, lymphocyte activation, $\mathrm{B}$ cell activation, alpha-beta $\mathrm{T}$ cell activation and the activation of immune response (Figure 4B). The GSEA analysis between high- and low-risk group of TCGA-SKCM cohort was also conducted. The low-risk group, which included the high level of GZMs, also shown the activated of immunocyte pathways and immune response pathways (Figure 4C-D).

Table 2. The coefficient of GZMscore and clinical features to the signature

\begin{tabular}{llllll}
\hline Parameters & coef & $\exp$ (coef) & se (coef) & $\mathrm{z}$ & P value \\
\hline GZMs score & -1.19228 & 0.30353 & 0.354172 & -3.366 & $7.62 \times 10^{-4^{*}}$ \\
Age & 0.01838 & 1.01855 & 0.005542 & 3.317 & $9.11 \times 10^{-4^{*}}$ \\
$\begin{array}{l}\text { Brelow's depth } \\
(>3 \mathrm{~cm} \text { vs. } \leq 3 \mathrm{~cm})\end{array}$ & 0.659245 & 1.933332 & 0.166378 & 3.962 & $7.42 \times 10^{-5^{*}}$ \\
$\begin{array}{l}\text { Stage } \\
\text { (III+IV vs. 0+I+II) }\end{array}$ & 0.5936 & 1.810494 & 0.16362 & 3.628 & $2.86 \times 10^{-4^{*}}$ \\
$\begin{array}{l}\text { Co-ef, co-efficient; Exp (co-ef), Expectation (co-ef); Se (co-ef), standard error (co-ef); } \\
\text { * P < 0.05. }\end{array}$
\end{tabular}

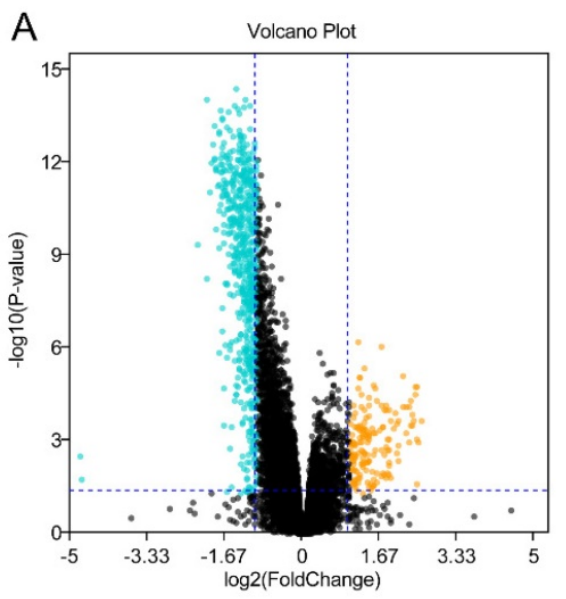

C

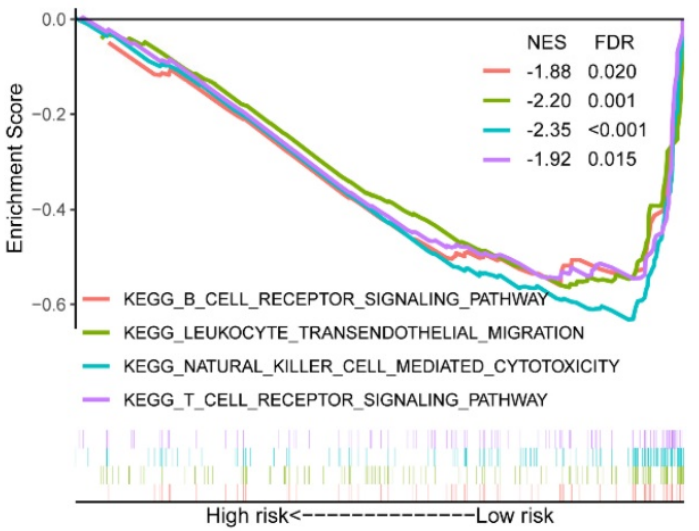

B

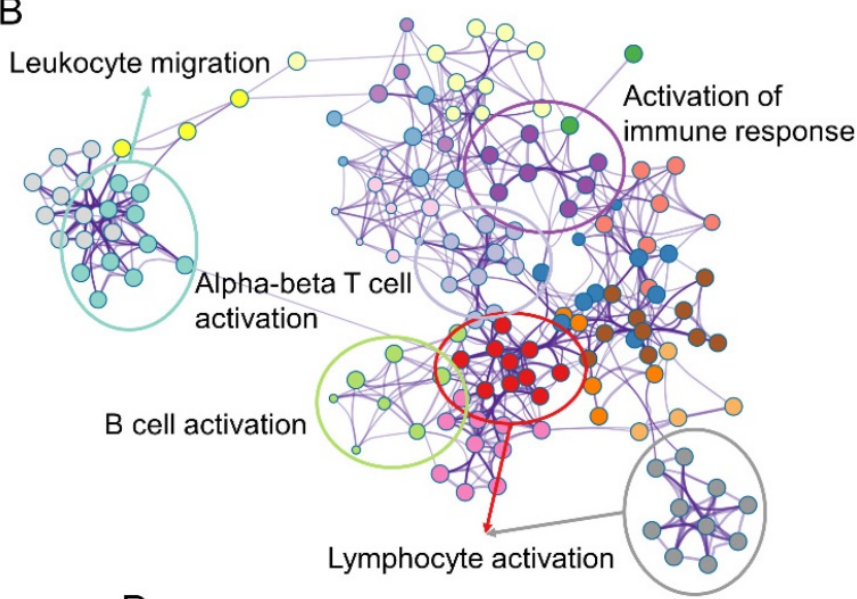

D

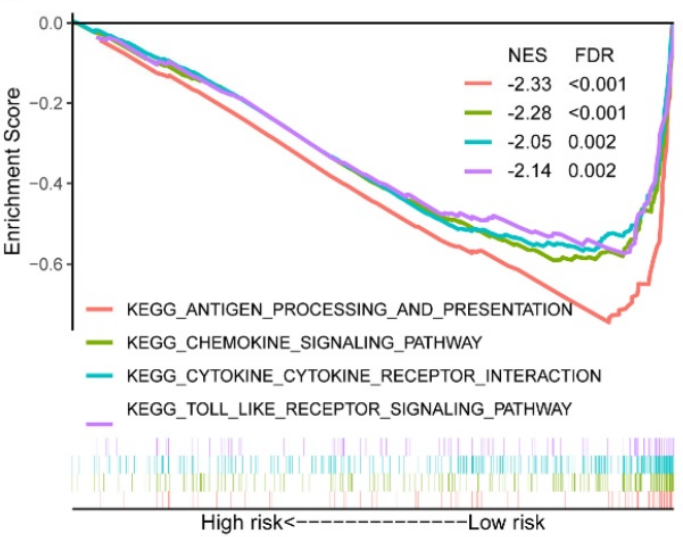

Figure 4. Different expression genes (DEGs) and signaling pathways between high- and low-risk groups in TCGA-SKCM cohort. (A) DEGs between high- and low-risk groups; (B) Signaling pathway annotation of decreased DEGs; (C-D) Activated signaling pathways in low-risk group evaluated by GSEA analysis. 

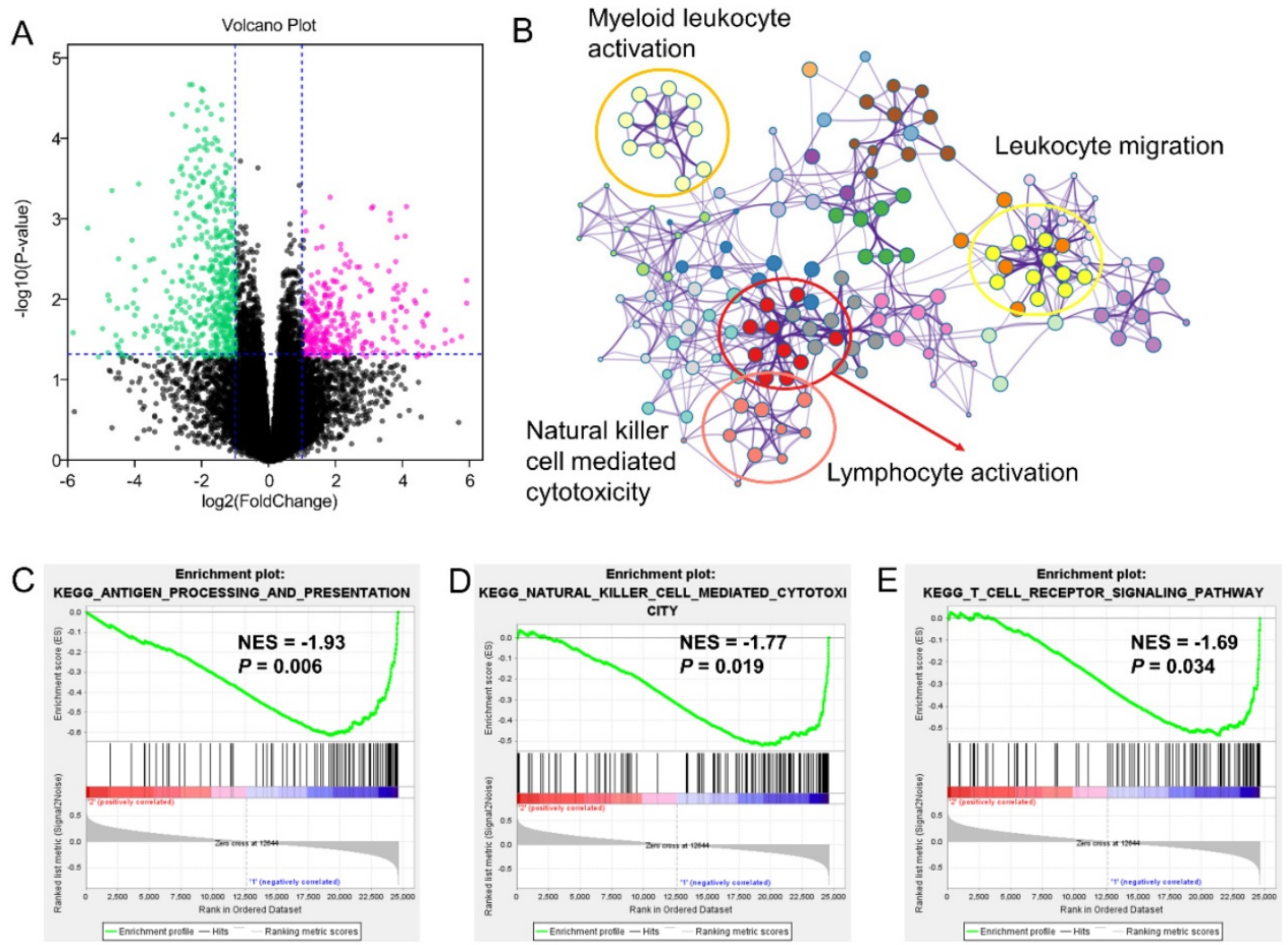

Figure 5. Different expression genes (DEGs) and signaling pathways between high- and low-risk groups in GSE65904 cohort. (A) DEGs between high- and low-risk groups; (B) Signaling pathway annotation of decreased DEGs; (C-E) Activated signaling pathways in low-risk group evaluated by GSEA analysis.

Table 3. The Distribution of clinical features among low- and high-risk group

\begin{tabular}{|c|c|c|c|c|c|c|}
\hline & \multicolumn{3}{|l|}{ TCGA-SKCM } & \multicolumn{3}{|l|}{ GSE65904 } \\
\hline & Low-Risk & High-Risk & & Low-Risk & High-Risk & \\
\hline Age, years old & $53.51 \pm 15.32$ & $64.44 \pm 13.65$ & $<0.001^{*}$ & $61.00 \pm 14.71$ & $66.28 \pm 13.84$ & 0.275 \\
\hline Gender & & & 0.092 & & & 0.74 \\
\hline Male & 112 & 97 & & 8 & 10 & \\
\hline Female & 56 & 72 & & 10 & 8 & \\
\hline Breslow's depth & & & $<0.001^{*}$ & & & $<0.001^{*}$ \\
\hline$\leq 3$ & 140 & 33 & & 17 & 5 & \\
\hline$>3$ & 28 & 136 & & 1 & 13 & \\
\hline Stage & & & $<0.001^{*}$ & & & - \\
\hline $0+\mathrm{I}+\mathrm{II}$ & 139 & 104 & & - & - & \\
\hline $\mathrm{III}+\mathrm{IV}$ & 29 & 65 & & 18 & 18 & \\
\hline GZMscore & $0.21 \pm 0.24$ & $0.03 \pm 0.20$ & $<0.001^{*}$ & $0.41 \pm 0.16$ & $0.14 \pm 0.22$ & $<0.001^{*}$ \\
\hline
\end{tabular}

The similar findings were generated from the GSE65904 as well. The decreased 549 genes in high-risk groups as compared with low-risk group was enriched in pathways of myeloid leukocyte activation, leukocyte migration, lymphocyte activation and natural killer cell-mediated cytotoxicity (Figure 5A-B). The GSEA analysis also pointed out the activation of antigen processing and presentation, natural killer cell-mediated cytotoxicity and $\mathrm{T}$ cell receptor signaling pathway in the low-risk GZMs group (Figure 5C-E). These results supported that GZMs positively associated with the activation of immune system and restrict the progression of $\mathrm{CM}$ in patients.

\section{Activated CD8+ cell negatively associated with the increased risk score}

The risk score of each patient reflected the combined predict effectiveness of GZMs and clinical information. With the results of the annotation of DEGs and GSEA analysis, we drew a line of risk score and immune signaling pathways. Furthermore, we compared the different infiltration of immunocytes between different risk groups. Based on the immunocytes infiltration prediction of CIBERSORT method, patients in the high-risk group shown an increased infiltration of resting NK cells, M0 and M2 macrophages, while the CD8+ $\mathrm{T}$ cell, activated 
memory CD4+ $\mathrm{T}$ cell, gamma delta $\mathrm{T}$ cell, activated NK cell and M1 macrophages infiltrated more in low-risk group (Figure 6A). Meanwhile, we also revealed that the risk score was negatively associated with the infiltration of most immunocytes calculated by ssGSEA of 28 specific types (Figure $6 \mathbf{6 B}$ ). The activated $\mathrm{CD} 8+\mathrm{T}$ cell shown the highest association with the risk score $(R=-0.48, P<0.01$, Figure $6 C)$, as well as the marker of CD8+ T cell, CD8A $(\mathrm{R}=-0.51, \mathrm{P}$ $<0.01$, Figure $6 \mathrm{D}$ ). The positive association of CD8+ T cell with GZMs was double confirmed by the TIMER, increased infiltration of CD8 $+\mathrm{T}$ cell correlated with the high expression of GZMA $(\mathrm{R}=0.721, \mathrm{P}<0.01)$, GZMB $(\mathrm{R}=0.605, \mathrm{P}<0.01)$, GZMH $(\mathrm{R}=0.606, \mathrm{P}<$ $0.01)$, GZMK $(\mathrm{R}=0.703, \mathrm{P}<0.01)$ and GZMM $(\mathrm{R}=$ $0.289, \mathrm{P}<0.01)$ (Figure S5). These findings suggested that the GZMs could inhibit the progress of CM throng the signaling pathways of activated CD8+ T cells.

A

type 官 Low 官 High

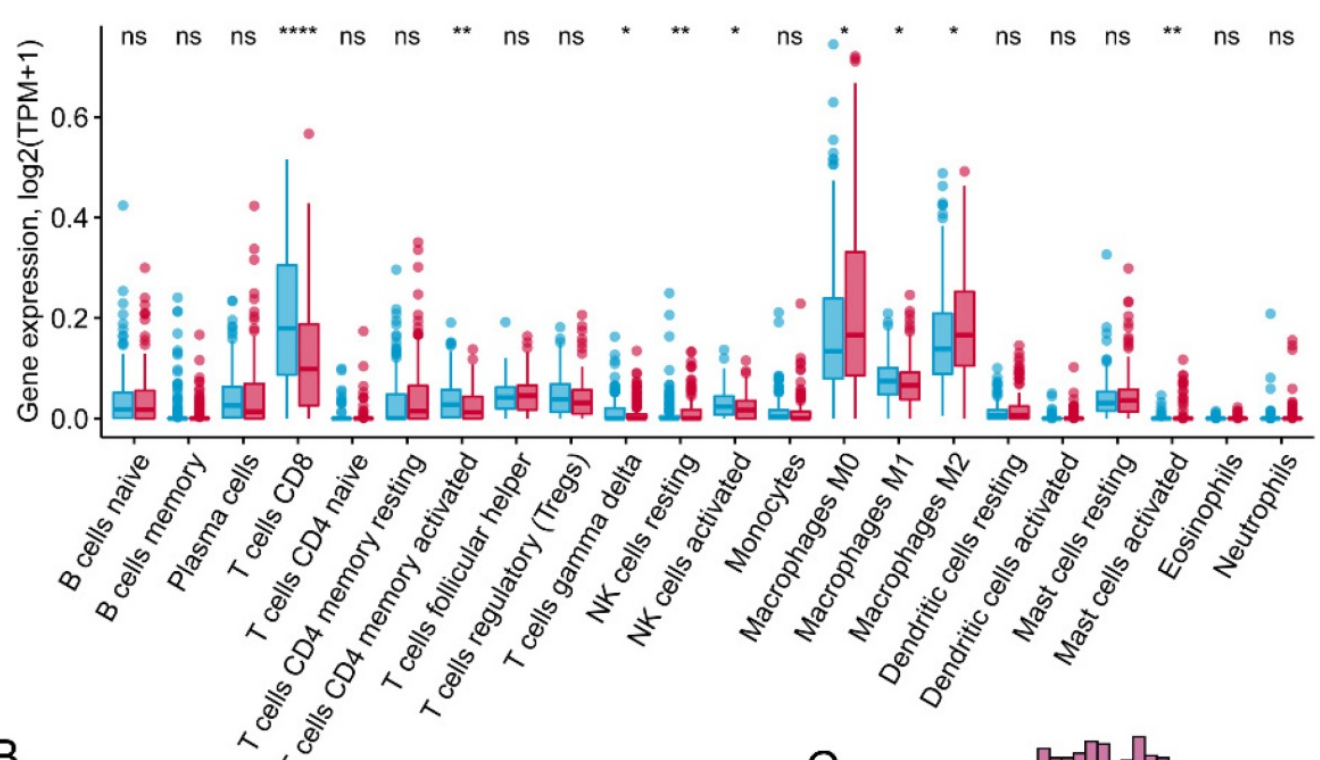

B

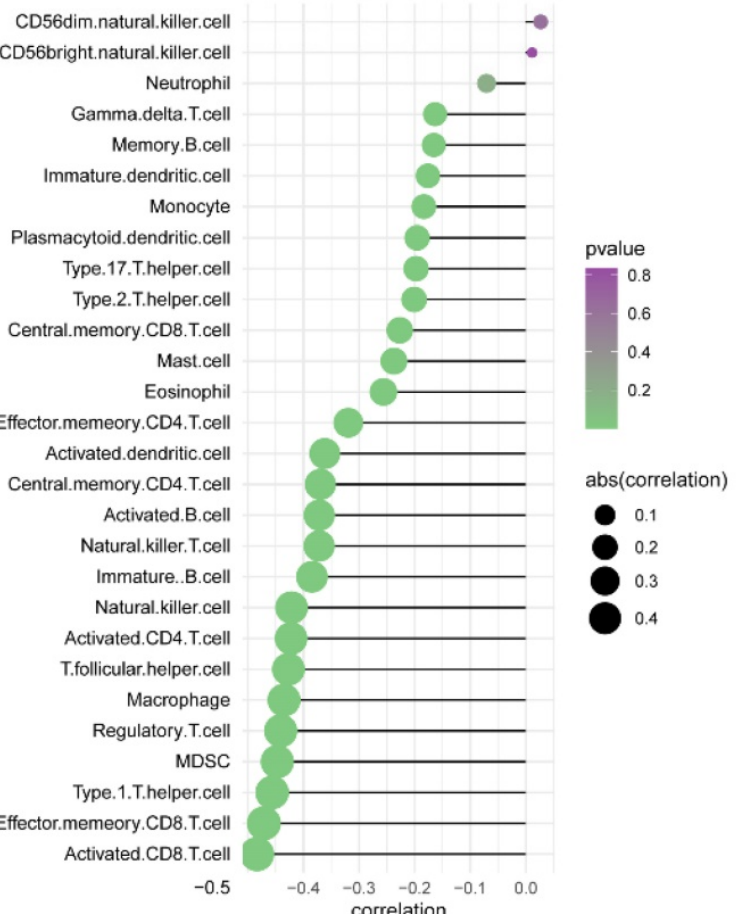

C
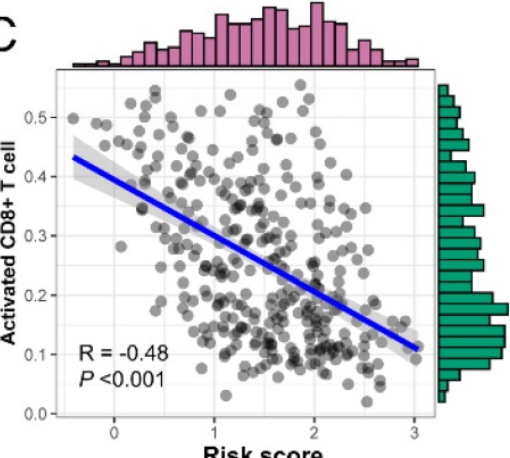

Risk score

$\mathrm{n}=337, \mathrm{r}=-0.51, P<0.001$

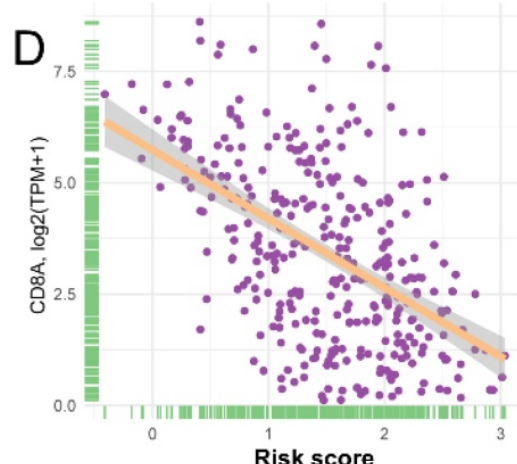

Risk score

Figure 6. Risk score is negatively associated with activated CD8+ T cells. (A) Different infiltration of 22 immunocytes calculated by CIBERSORT between risk groups; (B) Association of risk score with 28 immunocytes calculated by ssGSEA; (C) Risk score negatively associated with the abundance of activated CD8+ T cell; (D) Risk score negatively associated with the expression of CD8A. 

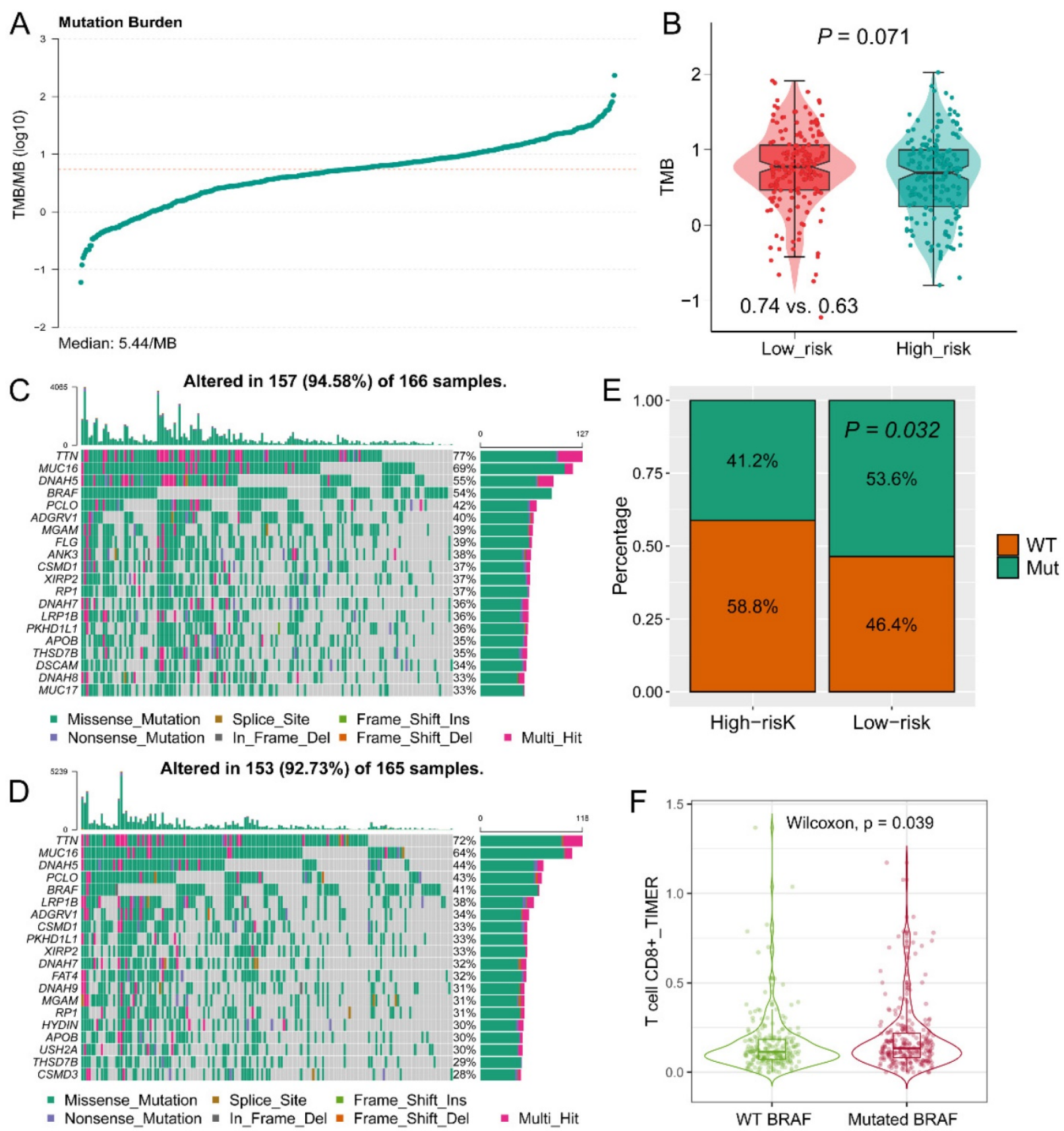

Figure 7. Tumor mutation burden (TMB) and mutant genes distribution in risk groups. (A) TMB score of patients in TCGA-SKCM cohort; (B) Different distribution of TMB in high- and low-risk groups; Distribution of mutant genes in low-risk (C) and high-risk (D) groups; (E) More BRAF mutation was observed in low-risk group; (F) Mutated BRAF leads the increased expression of BRAF mRNA.

\section{The landscape of tumor mutation among GZMs determined risk groups}

The TMB value of each patient in TCGA-SKCM cohort was calculated by $\mathrm{R}$ package "maftools" (Figure 7A). High TMB was observed in the low-risk group $(\mathbf{P}=\mathbf{0 . 0 7 1}$, Figure $7 \mathrm{~B})$, which is consistent with the findings of immune activation in low-risk group, cause the TMB might stimulate the activation of the tumor immune environment. The top 20 mutant genes in high- or low-risk groups were displayed in Figure 7C-D. BRAF mutation was observed more in the low- risk group $(\mathrm{P}=0.032$, Figure $7 \mathrm{E})$, and mutated $B R A F$ interacted with the increased infiltration of CD8+ T cell $(\mathrm{P}=0.039$, Figure $7 \mathrm{~F})$. Therefore, we speculated that the mutated BRAF in low-risk group could stimulate the activation of CD8+ T cells, and lead to a favorable OS.

\section{The potential responders filtered by the GZMs determined risk score}

The immunotherapy was widely used for CM patients, because the GZMs risk score is tightly associated with the immune infiltration. We predicted 
the potential benefit and attempted to find out the responders. The TIDE score for each patient was generated from the online tools (Figure 8A), patients in the low-risk group seemed to contain more responders for immunotherapy than highrisk group ( $40 \%$ vs. $19 \%, \mathrm{P}=0.029$, Figure $\mathbf{8 B}$ ). After compared the immunotherapy sensitive genes distribution with the anti-CTLA-4 and anti-PD-1 treated $\mathrm{CM}$ cohort, we revealed that patients in the low-risk group could benefit more from the therapy of anti-PD-1, but not anti-CTLA-4 (Bonferroni corrected $P=0.008$, Figure 8C).

\section{Discussion}

GZMs are the encoding genes of the pivotal components, granzymes, of immunocytes. GZMA is one of the key genes to quantify the cytolytic activity (CYT) of tumor cells [31], the CYT factor was widely used to measure the activation of immunocytes for the RNA sequence data [32, 33]. Shimizu et al. [34] reported that GZMA could activate the plasmacytoid dendritic cells and eliciting antigen-specific cytotoxic CD8+ T lymphocytes (CTLs). Rchiad et al. [35] reported the GZMA has a tumor suppressor function in $\mathrm{T}$. annulata-infected bovine host leukocytes and in human B-lymphomas. Yang et al. [36] reported that the HCA587 protein vaccine could eradicate the CM by increasing the expression of GZMB by CD4+ T cells, which is also a cooperate function of IFN- $\gamma$. Wu et al. [37] reposted that PFN, GzmA, GzmB, GNLY are the co-factors that affect the NK-extracellular vesicles mediated cytotoxicity to tumor cells, GzmA could inhibit the tumor cells through a caspase-independent death pathway. The prognostic value of GZMs family has not been illustrated till now.

In the current study, we evaluated the prognostic value of the five GZMs in CM patients. Decreased GZMs indicated the unfavorable OS, we revealed and confirmed the findings based on TCGA-SKCM cohort, GSE65904 cohort, GSE53118 cohort, GSE19234 cohort and GSE22153 cohort. Patients with the low-risk score determined by GZMs shown an activated status of immune-associated signaling, such as immune cell activation, immune cell migration and antigen presentation. Activated CD8+ T cells are the most impacted by GZMs. These results suggested us that the altered expression of GZMs could impact the progress of $\mathrm{CM}$ through CD8+ T cell-mediated immune response, $\mathrm{CM}$ patients with a poor prognosis might be caused by the exhausted of immune infiltration. In a further study, we predict the potential responders in high- and low-risk group. More immunotherapy responders

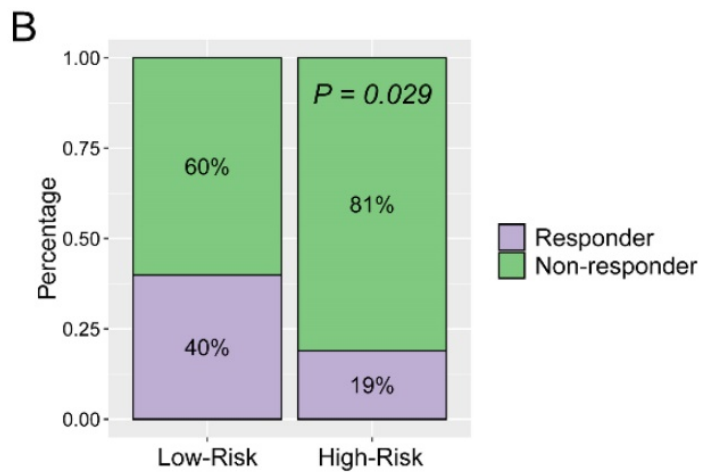

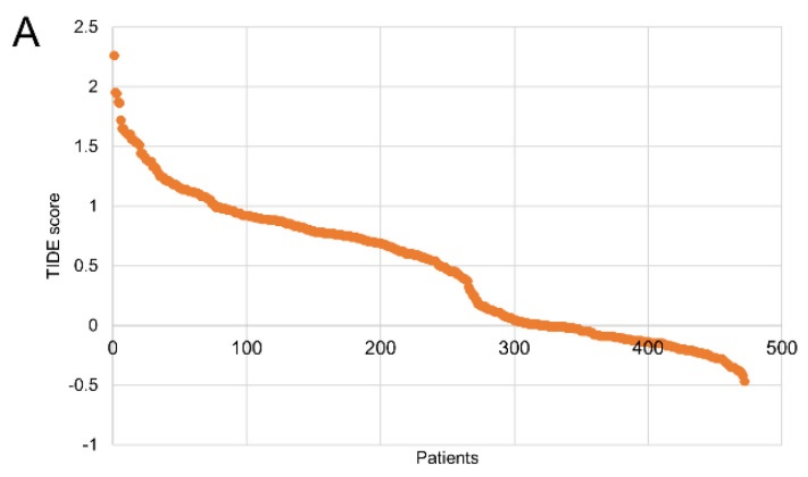

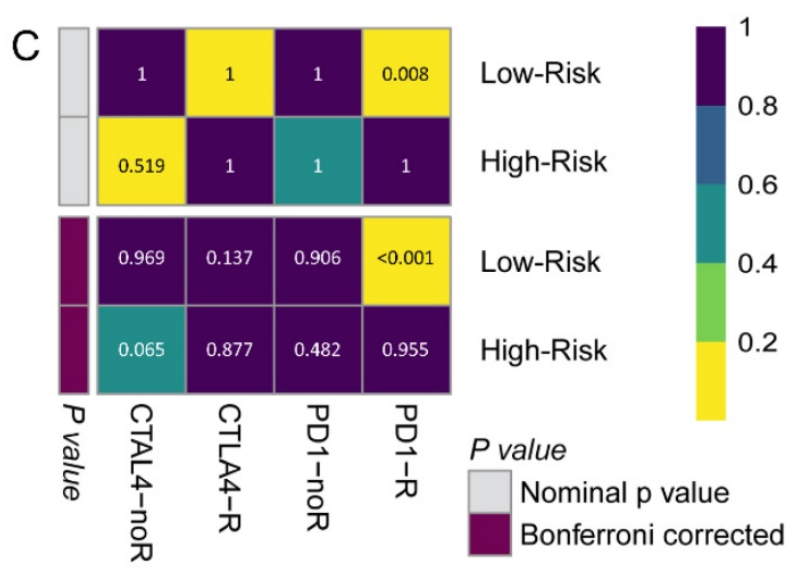

Figure 8. Low-risk group could benefit more from immunotherapy. (A) TIDE score of patients in TCGA-SKCM cohort; (B) More immunotherapy responders were observed in low-risk group; (C) Low-risk patients benefit more from anti-PD-1 therapy. 
were extracted in the low-risk groups, which mean with the high infiltration proportion, and in the contrary, high-risk group patients benefit less from the immunotherapy. Lymphocyte infiltration and abundant cytokines are the key features for the effective treatment of immune checkpoint inhibitors (ICI) therapy, the lack of $\mathrm{T}$ cell in the tumor microenvironment indicated the characteristics of a "cold tumor" [38]. In the subsequent predict of response to anti-CTAL4 and anti-PD-1 therapy, there is no doubt that the low-risk group patients could benefit more from the anti-PD-1 therapy.

What's more, we compared the distribution of mutated genes among two risk groups and revealed that BRAF mutation was observed more in the low-risk group, and mutated $B R A F$ interacted with the increased infiltration of CD8+ T cell. The mutation of $B R A F$ in $C M$ patients was widely studied. Davies et al. [39] reported that the somatic BRAF mutation was detected in about $66 \%$ malignant tumors, including $\mathrm{CM}$. Beuer et al. [40] concluded that age, anatomic site and degree of sunburn are the independent factors affect the frequency of BRAF mutation in $\mathrm{CM}$. Vemurafenib is a specific therapy for the $B R A F$ mutant $\mathrm{CM}$ patients, it could inhibit the ERK phosphorylation and causes the cell death of CM, which was approved by the US Food and Drug Administration [41]. In the current study, we revealed that patients in the low-risk group have a high rate of $B R A F$ mutation, therefore, these patients are the ideal ones to receive the vemurafenib treatment.

In summary, five GZMs are suppressors in CM tumorigenesis, GZMs could activate the immune response and prolong the OS of CM patients, patients' with the lower GZMscore could benefit more from anti-PD-1 therapy and vemurafenib treatment. Overexpression of GZMK could suppress the proliferation and migration, but not cell apoptosis in $\mathrm{CM}$ cell lines.

\section{Supplementary Material}

Supplementary figures.

http://www.medsci.org/v18p1657s1.pdf

\section{Acknowledgements}

This work was supported by Shenzhen Sanming Project (no. SZSM201812059) and Shenzhen Key Medical Discipline Construction Fund (No. SZXK040).

\section{Author contributions}

$\mathrm{WX}$ and ZJZ designed the study; WX, WXJ and $\mathrm{ZY}$ collected data; WX, and LK completed the statistics; WX drafted the manuscript; YB and ZJZ supervised the study. All authors read and approved the final manuscript.

\section{Data availability statement}

The datasets used and/or analyzed during the current study are available from the corresponding author on reasonable request.

\section{Competing Interests}

The authors have declared that no competing interest exists.

\section{References}

1. Schadendorf D, van Akkooi ACJ, Berking C, Griewank KG, Gutzmer R, Hauschild A, et al. Melanoma. Lancet. 2018; 392: 971-84.

2. Ossio R, Roldan-Marin R, Martinez-Said H, Adams DJ, Robles-Espinoza CD. Melanoma: a global perspective. Nat Rev Cancer. 2017; 17: 393-4.

3. Bray F, Ferlay J, Soerjomataram I, Siegel RL, Torre LA, Jemal A. Global cancer statistics 2018: GLOBOCAN estimates of incidence and mortality worldwide for 36 cancers in 185 countries. CA Cancer J Clin. 2018; 68: 394-424.

4. Yang DD, Salciccioli JD, Marshall DC, Sheri A, Shalhoub J. Trends in malignant melanoma mortality in 31 countries from 1985 to 2015 . Br J Dermatol. 2020

5. Volkovova K, Bilanicova D, Bartonova A, Letasiova S, Dusinska M. Associations between environmental factors and incidence of cutaneous melanoma. Review. Environ Health. 2012; 11 Suppl 1: S12.

6. Puckett Y, Wilson AM, Thevenin C. Cancer, Melanoma Pathology. StatPearls. Treasure Island (FL); 2020.

7. Potrony M, Badenas C, Aguilera P, Puig-Butille JA, Carrera C, Malvehy J, et al. Update in genetic susceptibility in melanoma. Ann Transl Med. 2015; 3: 210.

8. Robert C, Grob JJ, Stroyakovskiy D, Karaszewska B, Hauschild A, Levchenko E, et al. Five-Year Outcomes with Dabrafenib plus Trametinib in Metastatic Melanoma. N Engl J Med. 2019; 381: 626-36.

9. Larkin J, Ascierto PA, Dreno B, Atkinson V, Liszkay G, Maio M, et al. Combined vemurafenib and cobimetinib in BRAF-mutated melanoma. N Engl J Med. 2014; 371: 1867-76.

10. Hodi FS, O'Day SJ, McDermott DF, Weber RW, Sosman JA, Haanen JB, et al. Improved survival with ipilimumab in patients with metastatic melanoma. N Engl J Med. 2010; 363: 711-23.

11. Robert C, Long GV, Brady B, Dutriaux C, Maio M, Mortier L, et al. Nivolumab in previously untreated melanoma without BRAF mutation. N Engl J Med. 2015; 372: 320-30.

12. Anthony DA, Andrews DM, Watt SV, Trapani JA, Smyth MJ. Functional dissection of the granzyme family: cell death and inflammation. Immunol Rev. 2010; 235: 73-92.

13. Shimizu K, Sato Y, Shinga J, Watanabe T, Endo T, Asakura M, et al. KLRG+ invariant natural killer T cells are long-lived effectors. Proc Natl Acad Sci U S A. 2014; 111: 12474-9.

14. Lieberman J. Granzyme A activates another way to die. Immunol Rev. 2010; 235: 93-104.

15. Meng J, Liu Y, Guan S, Fan S, Zhou J, Zhang M, et al. The establishment of immune infiltration based novel recurrence predicting nomogram in prostate cancer. Cancer Med. 2019; 8: 5202-13.

16. Grabovska Y, Mackay A, O'Hare P, Crosier S, Finetti M, Schwalbe EC, et al. Pediatric pan-central nervous system tumor analysis of immune-cell infiltration identifies correlates of antitumor immunity. Nat Commun. 2020; 11: 4324.

17. Larsen AMH, Kuczek DE, Kalvisa A, Siersbaek MS, Thorseth ML, Johansen AZ, et al. Collagen Density Modulates the Immunosuppressive Functions of Macrophages. J Immunol. 2020; 205: 1461-72.

18. Trapani JA. Granzymes: a family of lymphocyte granule serine proteases. Genome Biol. 2001; 2: REVIEWS3014.

19. Wang L, Zhang K, Wu L, Liu S, Zhang H, Zhou Q, et al. Structural insights into the substrate specificity of human granzyme $\mathrm{H}$ : the functional roles of a novel RKR motif. J Immunol. 2012; 188: 765-73.

20. Zhou Y, Zhou B, Pache L, Chang M, Khodabakhshi AH, Tanaseichuk O, et al. Metascape provides a biologist-oriented resource for the analysis of systems-level datasets. Nat Commun. 2019; 10: 1523.

21. Subramanian A, Tamayo P, Mootha VK, Mukherjee S, Ebert BL, Gillette MA, et al. Gene set enrichment analysis: a knowledge-based approach for interpreting genome-wide expression profiles. Proc Natl Acad Sci U S A. $2005 ; 102$ : $15545-50$. 
22. Charoentong P, Finotello F, Angelova M, Mayer C, Efremova M, Rieder $\mathrm{D}$, et al. Pan-cancer Immunogenomic Analyses Reveal GenotypeImmunophenotype Relationships and Predictors of Response to Checkpoint Blockade. Cell Rep. 2017; 18: 248-62.

23. Mayakonda A, Lin DC, Assenov Y, Plass C, Koeffler HP. Maftools: efficient and comprehensive analysis of somatic variants in cancer. Genome Res. 2018; 28: 1747-56.

24. Hoshida Y, Brunet JP, Tamayo P, Golub TR, Mesirov JP. Subclass mapping: identifying common subtypes in independent disease data sets. PloS one. 2007; 2: e1195.

25. Jiang P, Gu S, Pan D, Fu J, Sahu A, Hu X, et al. Signatures of T cell dysfunction and exclusion predict cancer immunotherapy response. Nat Med. 2018; 24: 1550-8.

26. Lu X, Jiang L, Zhang L, Zhu Y, Hu W, Wang J, et al. Immune Signature-Based Subtypes of Cervical Squamous Cell Carcinoma Tightly Associated with Human Papillomavirus Type 16 Expression, Molecular Features, and Clinical Outcome. Neoplasia. 2019; 21: 591-601.

27. Chen PL, Roh W, Reuben A, Cooper ZA, Spencer CN, Prieto PA, et al. Analysis of Immune Signatures in Longitudinal Tumor Samples Yields Insight into Biomarkers of Response and Mechanisms of Resistance to Immune Checkpoint Blockade. Cancer discovery. 2016; 6: 827-37.

28. Goswami CP, Nakshatri H. PROGgeneV2: enhancements on the existing database. BMC Cancer. 2014; 14: 970.

29. Aguirre-Gamboa R, Gomez-Rueda H, Martinez-Ledesma E, Martinez-Torteya A, Chacolla-Huaringa R, Rodriguez-Barrientos A, et al. SurvExpress: an online biomarker validation tool and database for cancer gene expression data using survival analysis. PLoS One. 2013; 8: e74250.

30. Li T, Fu J, Zeng Z, Cohen D, Li J, Chen Q, et al. TIMER2.0 for analysis of tumor-infiltrating immune cells. Nucleic Acids Res. 2020; 48: W509-W14.

31. Rooney MS, Shukla SA, Wu CJ, Getz G, Hacohen N. Molecular and genetic properties of tumors associated with local immune cytolytic activity. Cell. 2015; 160: 48-61.

32. Gu X, Boldrup L, Coates PJ, Fahraeus R, Wang L, Wilms T, et al. High immune cytolytic activity in tumor-free tongue tissue confers better prognosis in patients with squamous cell carcinoma of the oral tongue. J Pathol Clin Res. 2019; 5: 240-7.

33. Romero JM, Grunwald B, Jang GH, Bavi PP, Jhaveri A, Masoomian M, et al. A Four-Chemokine Signature Is Associated with a T-cell-Inflamed Phenotype in Primary and Metastatic Pancreatic Cancer. Clin Cancer Res. 2020; 26: 1997-2010.

34. Shimizu K, Yamasaki S, Sakurai M, Yumoto N, Ikeda M, Mishima-Tsumagari C, et al. Granzyme A Stimulates pDCs to Promote Adaptive Immunity via Induction of Type I IFN. Front Immunol. 2019; 10: 1450 .

35. Rchiad Z, Haidar M, Ansari HR, Tajeri S, Mfarrej S, Ben Rached F, et al. Novel tumour suppressor roles for GZMA and RASGRP1 in Theileria annulata-transformed macrophages and human B-lymphoma cells. Cell Microbiol. 2020: e13255.

36. Yang W, Zhang W, Wang X, Tan L, Li H, Wu J, et al. HCA587 protein vaccine induces specific antitumor immunity mediated by CD4+ T-cells expressing granzyme B in a mouse model of melanoma. Anticancer Agents Med Chem. 2020

37. Wu CH, Li J, Li L, Sun J, Fabbri M, Wayne AS, et al. Extracellular vesicles derived from natural killer cells use multiple cytotoxic proteins and killing mechanisms to target cancer cells. J Extracell Vesicles. 2019; 8: 1588538 .

38. Bonaventura P, Shekarian T, Alcazer V, Valladeau-Guilemond J, Valsesia-Wittmann S, Amigorena S, et al. Cold Tumors: A Therapeutic Challenge for Immunotherapy. Front Immunol. 2019; 10: 168.

39. Davies H, Bignell GR, Cox C, Stephens P, Edkins S, Clegg S, et al. Mutations of the BRAF gene in human cancer. Nature. 2002; 417: 949-54.

40. Bauer J, Buttner P, Murali R, Okamoto I, Kolaitis NA, Landi MT, et al. BRAF mutations in cutaneous melanoma are independently associated with age, anatomic site of the primary tumor, and the degree of solar elastosis at the primary tumor site. Pigment Cell Melanoma Res. 2011; 24: 345-51.

41. Bollag G, Tsai J, Zhang J, Zhang C, Ibrahim P, Nolop K, et al. Vemurafenib: the first drug approved for BRAF-mutant cancer. Nat Rev Drug Discov. 2012; 11: 873-86. 